\title{
Review of: "Provider Confidence in Counseling Preconception, Pregnant, and Postpartum Patients Regarding COVID-19 Vaccination"
}

\author{
Fuqiang Cui ${ }^{1}$ \\ 1 Beijing Medical University
}

Potential competing interests: The author(s) declared that no potential competing interests exist.

Health care providers play the key role in counseling pregnant COVID-19 vaccination Tianshuo Zhao ${ }^{1,2}$, Fuqiang Cui ${ }^{1,2}$

1. Department of Laboratorial Science and Technology \& Vaccine Research Center, School of Public Health, Peking University, Beijing 100191, China

2. Global Center for Infectious Disease and Policy Research, Peking University, Beijing 100191, China

Corresponding authors

Fuqiang Cui, Professor,

Department of Laboratorial Science and Technology \& Vaccine Research Center

School of Public Health, Peking University

No. 38, Xueyuan Road, Haidian District, Beijing 100191, China.

Tel: +86-10-82801518; Email: cuifuq@bjmu.edu.cn

Pregnant women are considered a priority group for COVID-19 vaccination because of their increased risk of complications from COVID-19 infection although CDC recommendations are permissive about COVID-19 vaccine use in pregnancy, and there is no explicit recommendation that pregnant people should receive the vaccine as there is for the general adult population. Due to the absence of pregnancy specific vaccine evidence, and a lack of a clear directive to vaccinate all pregnant individuals, provider counseling will play a critical role in COVID-19 vaccine acceptance among pregnant people. ${ }^{1}$ This study explored the confidence of COVID-19 vaccine counseling among practitioners who provide care to pregnant women, understanding these factors will help to identify resources needed to improve healthcare provider confidence and improve counseling around shared decision making for vaccination for pregnant individuals. Given the limited evidence, focusing on the vaccination needs of pregnant women is an innovation of this study.

The study identified that most providers $(72.1 \%)$ reported the opinion that the benefits of the mRNA COVID 
19 vaccine in pregnancy outweigh the risks. It also identified a discrepancy between provider acceptance of vaccination as beneficial for pregnant individuals and their own comfort in engaging in conversations regarding the vaccine in pregnancy.

However, what caught my attention most was the vaccine hesitancy issue involved in this study. According to a survey in 16 countries, the majority of pregnant women refuse to receive COVID-19 vaccine because they are worried about the adverse effects on the infants and the lack of available data on safety and efficacy. ${ }^{2}$ Therefore, we would suggest that there are some works need to be done to identify the gap between the objective effect of vaccines (epidemiological evidence) and the subjective effect (selfperceived by pregnant women). The authors need to look at this issue further.

When we discuss the vaccine hesitancy, it involves many dimensions and fields, the author only chose vaccine hesitancy based on historic and continued racism and systemic injustice, we are wondering is there any other evidence to support these two factors? What are the predominant reasons for vaccine hesitancy in pregnant women? Despite providers' confidence in vaccines, there is a lack of strong advocacy from authoritative institutions to provide regulatory frameworks and guidelines for the inclusion of pregnant and lactating women in COVID-19 vaccination, as well as data on various safety profile registries. ${ }^{3,4}$ In addition to confidence and knowledge, this is crucial for the provider to make decisions. Although we agreed the overall findings, there are some issues beyond vaccine hesitancy need to be addressed. First, the main result of this study is the provider's self-efficacy (refers to a person's subjective judgment of whether he or she can successfully perform an accomplishment) confidence before consulting behavior, not the real consulting behavior. In other words, the confidence result may or may not be related to the counselling behavior that the provider ultimately produces. We think that's an issue that needs to be discussed. Second, knowledge is mentioned in the objective of this study, but we do not find the methods and results about knowledge of provider in the whole manuscript. Third, is there response bias in this study? Only 154 providers, mostly non-Hispanic white clinicians, filled out the questionnaire in this study. The author does not report the response rate or rejection rate, and whether the population in this study is representative of the population and can represent the real situation of the three hospitals. Fourth, as study suggested that all providers (92.2\%) reported that they always recommend the flu vaccine for pregnant individuals during flu season, and the majority (77.3\%) reported that they recommend the Tdap vaccine for pregnant individuals during every pregnancy. However, we cannot speculate this will contribute to the uptake of COVID-19 vaccine. Lastly, the results of this study are not all presented in the table and manuscript, such as the provider's belief about risk-benefit and the sources that survey respondents most commonly used to find information. All detailed information should be provided except the numbers shown in the manuscript. In particular, all the results of multivariable regression are not fully presented, readers need to know the variables included in the model. It is suggested that a complete table of study results be added to the supplemental materials.

\section{References}


1. Siegel MR, James KE, Jaffe E, L'Heureux MM, Kaimal AJ, Goldfarb IT. Provider Confidence in Counseling Preconception, Pregnant, and Postpartum Patients Regarding COVID-19 Vaccination. 2021: 2021.12.17.21267998.

2. Skjefte M, Ngirbabul M, Akeju O, et al. COVID-19 vaccine acceptance among pregnant women and mothers of young children: results of a survey in 16 countries. European journal of epidemiology 2021; 36(2): 197-211.

3. Garg I, Shekhar R, Sheikh AB, Pal S. COVID-19 Vaccine in Pregnant and Lactating Women: A Review of Existing Evidence and Practice Guidelines. Infectious disease reports 2021; 13(3): 685-99.

4. Stafford IA, Parchem JG, Sibai BM. The coronavirus disease 2019 vaccine in pregnancy: risks, benefits, and recommendations. American journal of obstetrics and gynecology 2021; 224(5): 484-95. 